\title{
Hall current effects in dynamic magnetic reconnection solutions
}

\author{
I. J. D. Craig and J. Heerikhuisen \\ Department of Mathematics, University of Waikato, Private Bag 3105, Hamilton, New Zealand \\ P. G. Watson \\ Center for Magnetic Reconnection Studies, Institute for Fusion Studies, University of Texas at Austin, \\ Austin, Texas 78712
}

(Received 21 February 2003; accepted 16 May 2003)

\begin{abstract}
The impact of Hall current contributions on flow driven planar magnetic merging solutions is discussed. The Hall current is important if the dimensionless Hall parameter (or normalized ion skin depth) satisfies $c_{H}>\eta$, where $\eta$ is the inverse Lundquist number for the plasma. A dynamic analysis of the problem shows, however, that the Hall current initially manifests itself, not by modifying the planar reconnection field, but by inducing a non-reconnecting perpendicular "separator" component in the magnetic field. Only if the stronger condition $c_{H}^{2}>\eta$ is satisfied can Hall currents be expected to affect the planar merging. These analytic predictions are then tested by performing a series of numerical experiments in periodic geometry, using the full system of planar magnetohydrodynamic (MHD) equations. The numerical results confirm that the nature of the merging changes dramatically when the Hall coupling satisfies $c_{H}^{2}>\eta$. In line with the analytic treatment of sheared reconnection, the coupling provided by the Hall term leads to the emergence of multiple current layers that can enhance the global Ohmic dissipation at the expense of the reconnection rate. However, the details of the dissipation depend critically on the symmetries of the simulation, and when the merging is "head-on" (i.e., comprises fourfold symmetry) the reconnection rate can be enhanced. (c) 2003 American Institute of Physics. [DOI: 10.1063/1.1590980]
\end{abstract}

\section{INTRODUCTION}

It seems likely that Hall currents play a significant role in magnetic merging solutions at realistic plasma resistivities (see Ref. 1 and the "GEM challenge"). Although Hall current effects are neglected in traditional magnetic reconnection models, ${ }^{2}$ there is mounting analytic and computational evidence that Hall effects are important for plasma resistivities typical of the solar corona. Reference 3, for example, provides numerical evidence that reconnection solutions lose their characteristic "current sheet" structure and become more "Petschek-type" due to the influence of Hall currents.

In general, Hall current contributions are expected to become significant when the dimensionless Hall coefficient $c_{H}$ satisfies

$$
c_{H}>\eta,
$$

where $\eta$ is the inverse Lundquist number for the plasma. For a typical coronal plasma with $c_{H} \simeq 10^{-7}$ and a collisional resistivity $\eta=\eta_{c} \simeq 10^{-14}$ this criterion is easily satisfied. Even enhanced anomalous resistivities $\eta \rightarrow \eta_{a} \simeq 10^{6} \eta_{c}$, which may occur in turbulent current sheets, ${ }^{4}$ are unlikely to undo this condition. The implication therefore, is that Hall currents should be important in virtually all cases of fast magnetic merging in the corona.

In a recent paper Craig and Watson ${ }^{5}$ point out that twoand three-dimensional, analytic magnetic reconnection solutions can be developed when Hall currents are included within a generalized Ohm's law for the plasma. This work provides a detailed treatment of planar current sheet models in which a perpendicular, nonreconnecting field-the "sepa- rator" component-is present. A key question is how reconnection rates and Ohmic energy release rates are influenced by the extra physical ingredients, namely, electron inertia and Hall current effects, that derive from the generalized Ohm's law. According to Ref. 5 the role of the inertial term is minor, but the Hall current can, under certain conditions, have dramatic consequences.

In fact condition (1) is found to influence mainly the development of the separator field; to affect the merging rate significantly a stronger condition $c_{H}^{2}>\eta$ is required. More specifically, Craig and Watson ${ }^{5}$ show that the planar merging problem is controlled by a dimensionless parameter $\kappa$ that must satisfy

$$
\kappa \simeq \frac{c_{H}^{2}}{\eta}>1
$$

for the merging rate to be appreciably affected. However, for large $\kappa$ the character of the solution completely changes and oscillations of wavelength $\sqrt{\kappa \eta} \sim c_{H}$ develop throughout the reconnection region. Thus, in addition to the primary current sheet that reconnects the magnetic field, an assemblage of secondary current sheets can emerge, and for $\kappa \sim 1$ this can lead to enhanced rates of Ohmic dissipation at the cost of reductions in the reconnection rate. This behavior contrasts with simulations of magnetically driven reconnection that report enhancements of the reconnection rate with increases in the Hall coefficient. ${ }^{6}$ A question that needs to be addressed, therefore, is whether this discrepancy is an artifact of the steady-state description of Craig and Watson or a general property of flow driven reconnection. 
The purpose of the present paper is to extend the work of Ref. 5 and revisit Hall current reconnection using a fully dynamic treatment of the governing equations. After introducing the Hall magnetohydrodynamics (Hall MHD) equations in Sec. II we then show in Sec. III that a timedependent analytic reduction is possible that isolates the role of the Hall current. In Sec. III we also illustrate how the Hall current provides a mechanism by which strong separator fields can be induced by the planar reconnecting field components. It is the effect of these induced axial fields that, for $\kappa>1$, dramatically modifies the characteristics of the merging, and in particular the quasi-one-dimensional current sheets of traditional magnetic merging. In Sec. IV we test the veracity of the analytic treatment using an incompressible Hall current code in periodic geometry. Our conclusions are presented in Sec. V.

\section{HALL CURRENT RECONNECTION EQUATIONS}

\section{A. Introduction}

We begin with the incompressible Hall MHD equations. If we then normalize field quantities according to the reference coronal values,

$$
\begin{aligned}
& B_{c}=10^{2} \mathrm{G}, \quad \ell_{c}=10^{9.5} \mathrm{~cm}, \\
& n_{c}=10^{9} \mathrm{~cm}^{-3}, v_{A}=10^{9} \mathrm{~cm} \mathrm{~s}^{-1},
\end{aligned}
$$

and times with respect to the Alfvén time $\tau_{A}=\ell_{c} / v_{A}$, we can derive a system of dimensionless equations that govern the evolution of the plasma.

In this formulation we can write the momentum equation as

$$
\frac{\partial \mathbf{v}}{\partial t}+(\mathbf{v} \cdot \nabla) \mathbf{v}=\mathbf{J} \times \mathbf{B}-\nabla p
$$

where $\mathbf{v}$ is the velocity, $\mathbf{B}$ is the magnetic field, $p$ is the total plasma pressure (electron plus ion), and $\mathbf{J}=\nabla \times \mathbf{B}$ is the current density. The generalized Ohm's law (ignoring electron inertia effects) is given by the equation,

$$
\mathbf{E}=-\mathbf{v} \times \mathbf{B}+\eta \mathbf{J}+c_{H}\left(\mathbf{J} \times \mathbf{B}-\nabla p_{e}\right),
$$

where $\mathbf{E}$ is the electric field and $p_{e}$ is the electron pressure (assumed to be a scalar). The dimensionless numbers,

$$
\eta=\frac{c^{2}}{4 \pi v_{A} \ell_{c} \sigma} \simeq 10^{-14.5}, \quad c_{H}=\frac{c}{\ell_{c} \omega_{p i}} \simeq 10^{-6.5}
$$

are coefficients appropriate for collisional coronal plasmas, where $c$ is the speed of light, $\sigma$ is the plasma conductivity, and $\omega_{p i}$ is the ion plasma frequency (in cgs units).

Faraday's law now allows us to write the evolution equation for the magnetic field

$$
\frac{\partial \mathbf{B}}{\partial t}=\nabla \times(\mathbf{v} \times \mathbf{B})-\nabla \times\left(\eta \mathbf{J}+c_{H} \mathbf{J} \times \mathbf{B}\right),
$$

where the $\mathbf{v}$ and $\mathbf{B}$ fields are constrained by

$$
\nabla \cdot \mathbf{v}=\nabla \cdot \mathbf{B}=0 .
$$

Note that energy can only be removed from the system by Ohmic dissipation. Since resistivities are always very small-they are unlikely to exceed $\eta_{a} \simeq 10^{-8}$ even if turbulent values ${ }^{2,4}$ are assumed-very steep gradients must develop in the B-field before a significant amount of the global energy can be resistively dissipated. More generally, the dissipation rate is so small that the magnetic field is almost completely frozen into the plasma. It follows that topological change by magnetic reconnection can only be effective in regions of high current density. Also note that the Hall coefficient $c_{H}$ (the normalized ion skin depth) satisfies both the inequalities $c_{H}>\eta$ and $c_{H}^{2}>\eta$, if a purely collisional value is assumed for the resistivity.

According to the analysis presented here, length scales may be so small, and the predicted current densities so high, that some noncollisional process of current limitation (e.g., turbulent resistivity) probably has to be introduced.

It should be recognized that the present Hall MHD equations contain several idealizations that seem inappropriate for a true coronal plasma. The assumption of incompressibility, for example, is a common expedient that allows a stream function representation for the velocity field, while avoiding the need for a detailed energy equation. Some justification is provided by side by side simulations of incompressible and compressible plasmas, which suggest that, as far as current sheet scalings with resistivity are concerned, no significant error is incurred through the assumption of incompressibility. This is hardly surprising since the sound travel time across a thin current sheet is far more rapid than typical magnetic merging time scales. However, we must also recognize that the inequalities $c_{H}>\eta$ and $c_{H}^{2}>\eta$ imply the limit of a strong magnetic field, which is not reflected in the isotropic form of the Ohm's law we adopt in Eq. (5). That is, for analytic and computational tractability we ignore anisotropies in the the conductivities and pressures and thermoelectric effects (see Spitzer, ${ }^{7}$ Chap. 2). In view of the many uncertainties that arise as a result of these assumptions, and the disturbingly high current densities that derive from the collisional treatment, our analysis is probably best regarded as a provisional estimate, rather than a definitive assessment of the role of Hall currents in reconnecting current layers.

\section{B. Planar field representation}

It is convenient for analytic and numerical purposes to examine so-called $2 \frac{1}{2} \mathrm{D}$ configurations, where $\mathbf{v}$ and $\mathbf{B}$ have all three field components but are only allowed to depend on two spatial coordinates. We can then adopt stream and flux function representations for the $\mathbf{v}$ and $\mathbf{B}$ fields,

$$
\begin{aligned}
& \mathbf{v}(x, y, t)=\nabla \phi \times \hat{\mathbf{z}}+W \hat{\mathbf{z}}, \\
& \mathbf{B}(x, y, t)=\nabla \psi \times \hat{\mathbf{z}}+Z \hat{\mathbf{z}},
\end{aligned}
$$

and introduce the Poisson bracket notation typified by

$$
[\psi, \phi]=\psi_{x} \phi_{y}-\phi_{x} \psi_{y},
$$

where subscripted variables denote partial derivatives. In components we have that $\mathbf{v}=\left(\phi_{y},-\phi_{x}, W\right)$ and $\mathbf{B}=\left(\psi_{y}\right.$, $\left.-\psi_{x}, Z\right)$.

From the curl of the momentum equation we note that the planar components of the velocity field are given by 


$$
\nabla^{2} \phi_{t}+\left[\nabla^{2} \phi, \phi\right]=\left[\nabla^{2} \psi, \psi\right],
$$

while

$$
W_{t}+[W, \phi]=[Z, \psi]
$$

determines the $z$-component. The third component of Ohm's law gives

$$
\psi_{t}+[\psi, \phi]=\eta \nabla^{2} \psi+c_{H}[\psi, Z],
$$

while the third component of the magnetic induction equation determines the separator field

$$
Z_{t}+[Z, \phi]=\eta \nabla^{2} Z+[W, \psi]+c_{H}\left[\nabla^{2} \psi, \psi\right] .
$$

This system completely determines the planar reconnection problem: it forms the basis of the analytic treatment given below, and the numerical treatment of a "closed" periodic geometry presented in Sec. IV.

A key property of the Hall term is that it nonlinearly couples the separator field $Z(x, y, t)$ to the otherwise autonomous planar field components. This coupling is particularly strong in the separator field equation (14), which involves third order derivatives of the flux function. The development of a strong separator field is clearly manifested in the planar components of the current density

$$
\mathbf{J}=\left(Z_{y},-Z_{x},-\nabla^{2} \psi\right) \text {. }
$$

In traditional reconnection models it is only the axial current $-\nabla^{2} \psi \hat{\mathbf{z}}$, generated by the reconnecting planar field, that contributes to the Ohmic dissipation rate,

$$
W_{\eta}=\eta \int J^{2} d V \text {. }
$$

Given that Hall-induced separator currents increase the magnitude of the current density, they have the potential to enhance the overall rate of magnetic energy release. We also point out that since $W_{\eta}$ is measured in units of $B_{c}^{2} v_{A} \ell_{c}^{2} / 8 \pi$ $\simeq 4 \times 10^{30} \mathrm{erg} / \mathrm{s}$, a value of $W_{\eta} \simeq 10^{-3}$ for the nondimensionalized problem corresponds to a sizable flare yielding approximately $10^{30}$ ergs over $100 \mathrm{~s}$.

\section{ANALYTIC TREATMENT OF HALL CURRENT RECONNECTION}

\section{A. Analytic form of the solution}

It is a remarkable fact that two and three-dimensional reconnection solutions based on a generalized Ohm's law can be developed analytically in the same manner as purely resistive solutions. ${ }^{5}$ The simplest approach for separator reconnection is to analyze systems (11)-(14) in terms of the Craig and Henton ${ }^{8}$ potentials,

$$
\phi=-\alpha x y+f(x, t), \quad \psi=\beta x y+g(x, t),
$$

making the axial field replacements

$$
W \rightarrow W(x, t), \quad Z \rightarrow Z(x, t) .
$$

These forms are consistent with the development of a onedimensional current sheet aligned with the $y$-axis; in fact they provide the leading order terms in any quasi-onedimensional description of magnetic merging developed by taking moments in $y$ (compare Ref. 9).
More physically, the potentials $f$ and $g$ determine planar disturbance fields that are advected by the background flow $\alpha x y$. To achieve current localization at the origin the constraint $0 \leqslant|\beta|<\alpha$ must be imposed. This condition ensures that the flow amplitude $\alpha$ is strong enough to localize the disturbance function $g(x, t)$ against the tendency of shear magnetic waves (for $|\beta|>0$ ) to propagate energy out of the reconnection region. ${ }^{10,11}$ In particular, because $\beta$ determines the curvature of field lines entering the current sheet it must be nonvanishing to obtain reconnection-otherwise there is only magnetic annihilation of straight field lines supported by a simple stagnation point flow. ${ }^{12}$ If $\beta$ is nonzero, then reconnection occurs in the high current region near the origin, and the merging is sustained by the advection of magnetic flux washed through the inflow boundaries $x= \pm 1$. The geometry is "open" in that flux entering through the sidewalls and reconnected at the origin, can be ejected by a magnetic sling shot through the top and bottom boundaries $y= \pm 1$.

Substituting the above forms into the reconnection system gives equations for the velocity field components,

$$
\begin{aligned}
& \frac{d f}{d t}=-2 \alpha f+\beta x g_{x}-2 \beta g, \\
& \frac{d W}{d t}=\beta x Z_{x},
\end{aligned}
$$

together with the magnetic field components,

$$
\begin{aligned}
& \frac{d g}{d t}=\beta x f_{x}+\eta g_{x x}-c_{H} \beta x Z_{x}, \\
& \frac{d Z}{d t}=\beta x W_{x}+\eta Z_{x x}+c_{H} \beta x g_{x x x},
\end{aligned}
$$

where we have introduced the Lagrangian derivative,

$$
\frac{d}{d t}=\frac{\partial}{\partial t}+\mathbf{v} \cdot \nabla=\frac{\partial}{\partial t}-\alpha x \frac{\partial}{\partial x} .
$$

Note that Eq. (19) is an integrated form of the momentum equation, valid under the assumption that $f(x, t)$ is an even function of $x$.

In exploring the role of the Hall current Craig and Watson $^{5}$ considered only the steady-state limit of these equations in which $f=-\beta g / \alpha$ and $W=-\beta Z / \alpha$. Our main concern in this section is to give a more general dynamic treatment, valid during the initial development of the reconnection current sheet, although we shall briefly revisit the steady-state model later in Sec. III D.

\section{B. The resistive current sheet in the absence of Hall currents}

We consider first of all the simplest case $c_{H}=0$. In this case the planar fields evolve independently of the perpendicular components and to satisfy (20) and (22) we can choose

$$
Z=Z_{0}=\text { const }, \quad W=0 .
$$



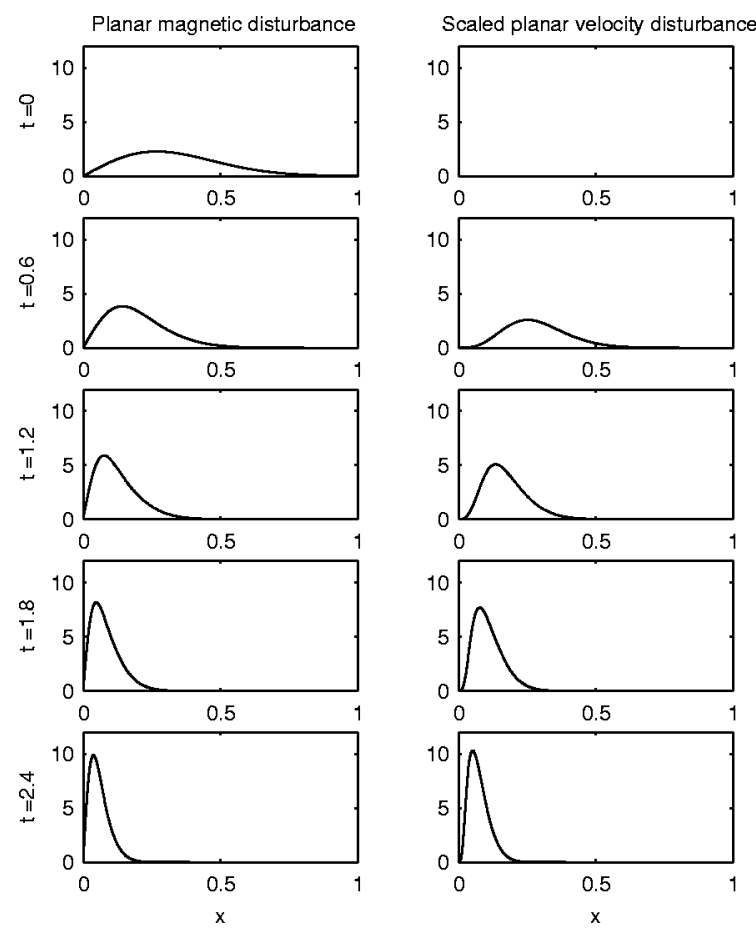

FIG. 1. Profiles of the planar magnetic disturbance field (left panels) and scaled velocity disturbance field (right panels) at equal time intervals from $t=0$ to $t=2.4$, showing the localization of the initial pulse. The wave peak moves from right to left as time increases. At later times the corresponding velocity field, when scaled by $-\alpha / \beta$, almost exactly mirrors the magnetic disturbance. Parameters used are $\eta=0.0005, \alpha=1, \beta=0.4, c_{H}=\kappa=0$.

Of particular interest is how a large scale magnetic disturbance located in the outer field is advected and localized by the background flow $\alpha x y$ as it approaches the origin. That is, we are interested in how a global field disturbance with the initial form,

$$
g(x, 0)=G(x) \text { with } f(x, 0)=0,
$$

leads to the formation of a strong current layer at the origin.

A formal argument based on the Klein-Gordon equation that governs the field evolution in the ideal limit ${ }^{10,11}$ shows that even if the velocity potential $f(x, t)$ is negligible initially, it cannot remain so during the advection of the disturbance field. In fact the velocity disturbance in the outer field equalizes, on an Alfvénic time scale, to the level $f \simeq-\beta g / \alpha$. By contrast the magnetic potential localizes relatively slowly.

Figure 1 shows the localization phase for an initial magnetic pulse of the form $g(x, 0)=G(x)=\exp \left(-7 x^{2}\right)$. Although the velocity potential $f$ is zero initially, it builds up within an Alfvén time to the amplitude $\beta g(x, t) / \alpha$, and from then on essentially mirrors the growth and localization of the magnetic field. The velocity field induced by the localization adds a transient, shearing component to the global background flow $\phi=-\alpha x y$. In this figure we have plotted the disturbance fields $\left(\alpha f_{x} / \beta\right.$ for the scaled velocity field and $-g_{x}$ for the magnetic field) rather than the disturbance potentials $f$ and $g$.

The evolution displayed in Fig. 1 can be understood by an informal argument based on the behavior of the function,

$$
h(x, t)=f+\frac{\beta}{\alpha} g .
$$

Substituting for $f$ in (19) and (21) we obtain

$$
\begin{aligned}
& h_{t}-\alpha^{+} x h_{x}+2 \alpha h=\frac{\beta}{\alpha}\left(\alpha^{-} x g_{x}+\eta g_{x x}\right), \\
& g_{t}-\alpha^{-} x g_{x}=\eta g_{x x}+\beta x h_{x},
\end{aligned}
$$

where we have introduced

$$
\alpha^{+}=\frac{\alpha^{2}+\beta^{2}}{\alpha}, \quad \alpha^{-}=\frac{\alpha^{2}-\beta^{2}}{\alpha} .
$$

The important point is that since $\alpha^{-}<\alpha^{+}$the advection of the magnetic field occurs more slowly than the localization of $h$. For the case of sufficiently small $\alpha^{-}$, the right-hand side of (27) can be neglected - the resistive term is negligible until the current sheet builds up-with the result that

$$
h \simeq h_{0}(\zeta) \exp (-2 \alpha t), \quad \zeta=x \exp \left(\alpha^{+} t\right),
$$

where $h_{0}$ is the initial $h$ profile. Clearly $h(x, t)$ is a wave that both propagates inward and decays to zero on a fast Alfvénic time scale, hence it effectively sets up the disturbance velocity potential at the level $f \simeq-\beta g / \alpha$. After the equalization phase the magnetic field evolves according to (28) with $h$ $\equiv 0$, that is

$$
g_{t}-\alpha^{-} x g_{x}=\eta g_{x x} .
$$

We can now answer the question: How long does it take before significant dissipation sets in? First, note that the solution in the absence of resistivity, namely,

$$
g(x, t)=G(\xi), \quad \xi=x \exp \left(\alpha^{-} t\right),
$$

will hold during the initial advective phase. By fixing a point on the trailing edge of the wave profile, say $\xi_{p}=1$, the localization of the wave is evident from the expression $x_{p}$ $=\exp \left(-\alpha^{-} t\right)$. However, as the field gradients build up the resistive term will begin to assert its influence and solution (32) will breakdown. The time of the breakdown can be estimated by comparing $\eta g_{x x}$ with the magnitude of the advective term $\alpha^{-} x g_{x}=\alpha^{-} \xi g_{\xi}$, under the assumption that the derivatives of $G(\xi)$ are of order unity (as befits a global initial disturbance). This suggests that the current sheet is set up on the localization time scale

$$
\tau_{S}=\frac{1}{2 \alpha^{-}} \ln \frac{\alpha^{-}}{\eta} .
$$

The trailing edge of the wave at this time, namely, $x_{p}$ $=\exp \left(-\alpha^{-} \tau_{S}\right)$, now identifies the outer edge for the current layer,

$$
x_{S}=\sqrt{\frac{\eta}{\alpha^{-}}} .
$$

It follows that the current sheet is very thin, $x_{s}<10^{-7}$, corresponding to a layer of a few hundred centimeters or less. As discussed in Sec. IIIE, the current densities implied by this result seem too large to be physically plausible. 

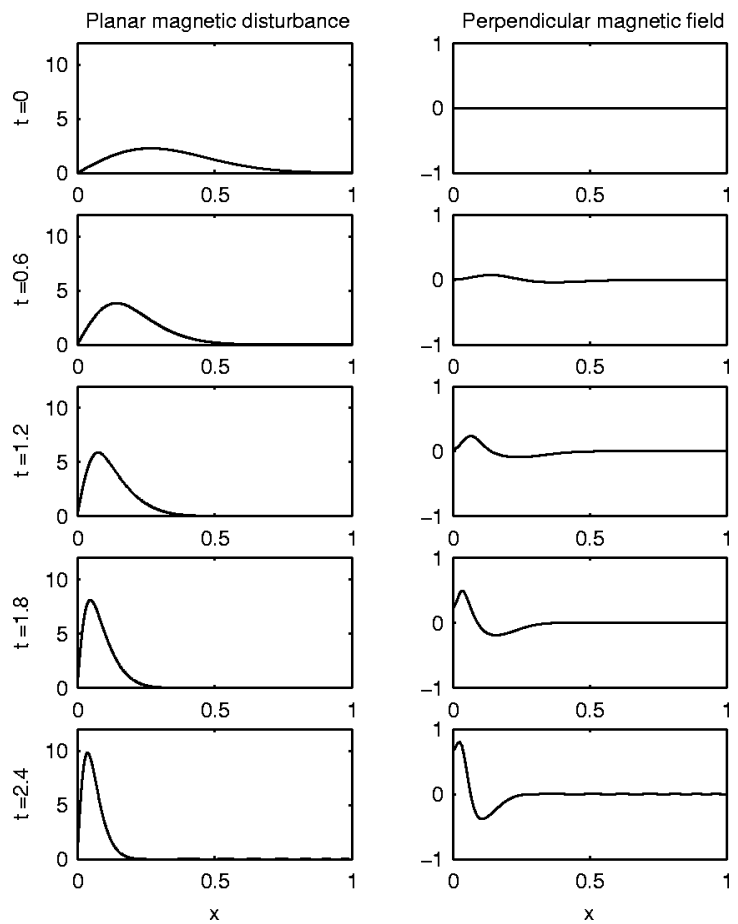

FIG. 2. Profiles of the planar magnetic disturbance field (left) and the perpendicular magnetic field $Z$ (right) for a modest value of the Hall parameter $c_{H}=0.01 \quad(\kappa \simeq 0.04)$ at equal time intervals from $t=0$ to $t=2.4$. The initial condition and all other parameters are the same as for Fig. 1. Note that the left-hand panels for this run are almost identical to those for Fig. 1. The main difference between this simulation and the purely resistive calculation is the evolution of a significant perpendicular field.

\section{Influence of the Hall current}

In line with the previous argument let us assume that the disturbance velocity fields have been equalized according to $f \simeq-\beta g / \alpha$ and $W \simeq-\beta Z / \alpha$. If we retain the Hall terms the system to be analyzed is now given by

$$
\begin{aligned}
& g_{t}-\alpha^{-} x g_{x}=\eta g_{x x}-\beta c_{H} x Z_{x}, \\
& Z_{t}-\alpha^{-} x Z_{x}=\eta Z_{x x}+\beta c_{H} x g_{x x x},
\end{aligned}
$$

and we shall specialize to the case where the separator field $Z$ is initially zero. If the Hall coefficient is nonzero we cannot have $Z=W=0$ for all time unless the merging is strictly head-on (that is $\beta=0$ ).

It is convenient to introduce the "co-moving" coordinates

$$
\tau=t \quad \xi=x \exp \left(\alpha^{-} t\right),
$$

so that

$$
\begin{aligned}
& g_{\tau}=\eta \exp \left(2 \alpha^{-} \tau\right) g_{\xi \xi}-\beta c_{H} \xi Z_{\xi}, \\
& Z_{\tau}=\eta \exp \left(2 \alpha^{-} \tau\right) Z_{\xi \xi}+\beta c_{H} \exp \left(2 \alpha^{-} \tau\right) \xi g_{\xi \xi \xi} .
\end{aligned}
$$

The equation for $Z$ already suggests that $\beta c_{H} \sim \eta$ is a necessary condition for the importance of the Hall current. By taking $c_{H}=\eta=0$ we immediately recover the advection solution $g(\xi, \tau) \simeq G(\xi)$ of the previous section.

Figure 2 shows plots of the planar and perpendicular magnetic fields for a run with a modest value of the Hall parameter. We have used the same parameters as in Fig. 1 and set $c_{H}=0.01$ (corresponding to a value $\kappa \simeq 0.04$ ). For this small value of $c_{H}$ the planar magnetic field (shown in the time slices on the left) is virtually identical to the purely resistive solution shown in Fig. 1, however, the perpendicular magnetic field has grown appreciably, unlike the resistive case where it remains zero throughout the simulation.

To understand the growth in the perpendicular field we can take the ideal approximation $g(\xi, \tau)=G(\xi)$ and substitute it into (39) on the assumption that $\beta c_{H} \gg \eta$ and that $Z$ $=0$ initially. The approximate solution for $Z$, namely,

$$
Z(\xi, \tau) \simeq \frac{\beta c_{H}}{2 \alpha^{-}} \xi G_{\xi \xi \xi}\left(\exp \left(2 \alpha^{-} \tau\right)-1\right)
$$

illustrates how a rapid $\exp \left(2 \alpha^{-} \tau\right)$ growth in the separator field is induced by the advection of the planar field. This growth essentially mimics the time development of the planar current density $g_{x x}=\exp \left(2 \alpha^{-} \tau\right) G_{\xi \xi}$. According to the computational merging experiments of Sec. IV, this result seems to be a general property of Hall current reconnection.

We are interested in whether the growth in $Z$ can interfere with the planar merging rate. If we compare the Hall term in (35) based on (40) with the advection term $\alpha^{-} x g_{x}$ $=\alpha^{-} \xi g_{\xi}$, then we deduce that the Hall term is effective for times greater than

$$
\tau_{H}=\frac{1}{\alpha^{-}} \ln \frac{\alpha^{-}}{\beta c_{H}} .
$$

We require $\tau_{H}<\tau_{S}$ [as defined by (33)] if the Hall term is to modify the current sheet formation. This gives

$$
\kappa \equiv \frac{\beta^{2} c_{H}^{2}}{\eta \alpha^{-}}>1
$$

which, to within factors of order unity, is just condition (2) cited in the Introduction.

Figure 3 shows a run for a large value of the Hall parameter $c_{H}=0.15$, corresponding to $\kappa \simeq 8$.6. In this limit the solution undergoes fundamental changes. Like the purely resistive solution there is an initial localization phase where the planar field obeys the approximate ideal solution and the perpendicular field is vanishingly small. However, once the initial implosion is halted an outgoing whistler wave propagates into the outer field setting up large-scale oscillations. Notice also the broadening and reduction in amplitude of the primary current sheet (compare the left-hand panels for $t$ $=2.4$ in Figs. 2 and 3). This reduction in the current (slope) at the origin implies a reduction in the reconnection rate.

\section{Quasisteady merging}

To obtain further insight into the problem for $\kappa \gg 1$ it is instructive to revisit the steady-state treatment of Craig and Watson. ${ }^{5}$ The equations we need to solve are (35) and (36) under the replacements $g_{t} \rightarrow \mathcal{E}, Z_{t} \rightarrow 0$, that is,

$$
\begin{aligned}
& \mathcal{E}-\alpha^{-} x g^{\prime}=\eta g^{\prime \prime}-\beta c_{H} x Z^{\prime}, \\
& -\alpha^{-} x Z^{\prime}=\eta Z^{\prime \prime}+\beta c_{H} x g^{\prime \prime \prime},
\end{aligned}
$$

where $\mathcal{E}$ is the flux transfer rate of the planar field. Here we provide an informal treatment based on a simple matching 

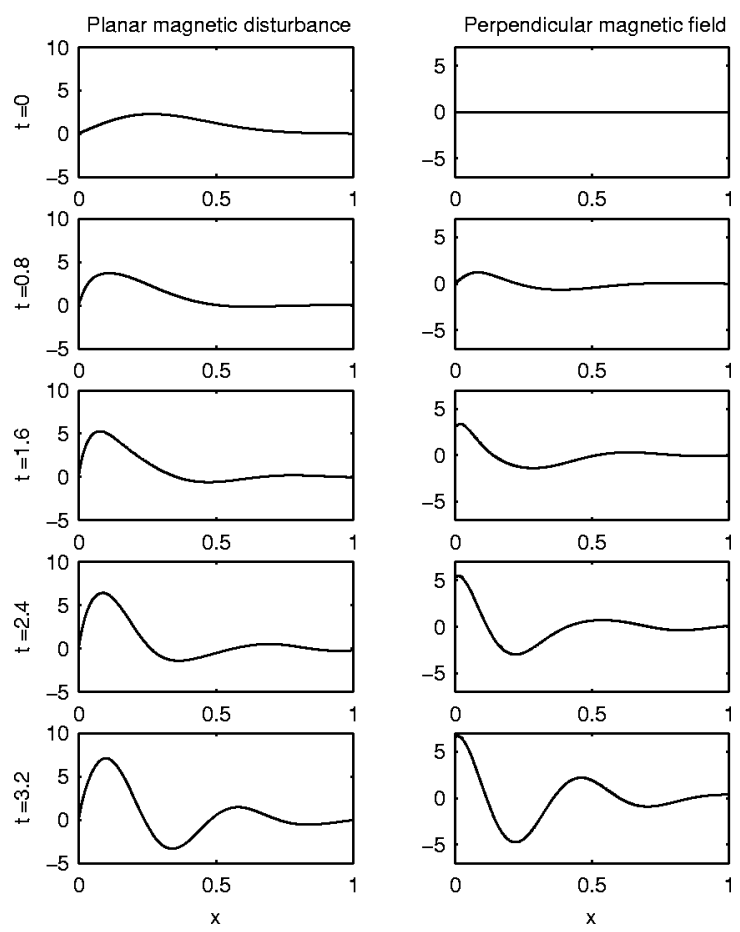

FIG. 3. Profiles of the planar magnetic disturbance field (left) and the perpendicular magnetic field $Z$ (right) for a large value of the Hall parameter $c_{H}=0.15$ at equal time intervals from $t=0$ to $t=3.2$. The initial condition and all other parameters are the same as for Fig. 1. This simulation now shows significant changes in the planar magnetic field. After the initial localization phase an outgoing whistler wave is generated that traverses the outer field, setting up an oscillatory wave pattern. This large-scale oscillation is evident in both the planar and perpendicular fields.

argument, valid for small $\eta$ and $\mathcal{E}$ and large $c_{H}$. A more indepth analysis is given by Craig and Watson, ${ }^{5}$ who also provide detailed numerical examples.

First note that at the origin we must have

$$
g^{\prime \prime}(x=0)=\frac{\mathcal{E}}{\eta} \text {. }
$$

Next we let $\eta$ and $\mathcal{E}$ vanish in Eqs. (43) and (44) - letting $\eta \rightarrow 0$ is consistent with $\kappa \gg 1$, but we must verify that $\mathcal{E}$ is small a posteriori. The solution of this system with the symmetries we require is then given by

$$
g^{\prime}=A \sin \left(\frac{\alpha^{-}}{\beta c_{H}} x\right), \quad Z=-A \cos \left(\frac{\alpha^{-}}{\beta c_{H}} x\right) .
$$

We can now obtain an approximate solution to the system in which $\eta$ and $\mathcal{E}$ are small by choosing the amplitude $A$ so that this solution satisfies the inner condition (45). Doing this we find

$$
A=\frac{\mathcal{E}}{\eta}\left(\frac{\beta c_{H}}{\alpha^{-}}\right) .
$$

For this solution to be valid we need to check that $\mathcal{E}$ is small. This is clearly true if the wave amplitude $A$ is fixed, as $\mathcal{E}$ $\sim \eta / c_{H}$, which does indeed tend to zero if $\kappa \gg 1$. Comparing this approximate solution with numerical solutions of Eqs. (43)-(44) we find that it is extremely accurate for $\kappa \gtrsim 10$.
The key implication of the steady-state analysis for $\kappa$ $\gg 1$ is the development of oscillatory behavior over the entire reconnection region on the length-scale,

$$
L_{H}=2 \pi \frac{\beta c_{H}}{\alpha^{-}}=2 \pi\left(\frac{\kappa \eta}{\alpha^{-}}\right)^{1 / 2}
$$

corresponding to a standing whistler wave. This implies that secondary current layers may be present in the merging solution that are quite different in character from the primary current sheet that provides the reconnection. Note also, that the flux transfer rate $\mathcal{E}$ is very small, $\mathcal{E} \simeq \eta / c_{H} \simeq 10^{-8}$. Although the Ohmic dissipation rate of each current layer is also weak, the overall dissipation rate may still be significant due to the additive effect of all such layers, a point confirmed numerically in Sec. IV C below.

\section{E. Reconnection and Ohmic dissipation rates}

A central question is how does the presence of the Hall term alter the flux reconnection and energy dissipation rates of the solution? By invoking the previous analytic results we can make some general comments based on the changing nature of the solution as $\kappa$ is increased.

In the regime of small $\kappa$ we expect the planar field to be largely unaffected by the Hall term and hence the reconnection rate should also be unchanged. The development of perpendicular magnetic fields should, however, give rise to an increase in the power dissipation rate due to the presence of additional currents that now flow in the plane.

For large $\kappa$ the picture alters dramatically. An outgoing whistler wave (see Fig. 3) now establishes large-scale global oscillations of the field. The effect of the wave is to transport flux away from the primary sheet at the origin back into the outer field, thereby reducing the reconnection rate. Also, as the oscillations become broader with increasing $\kappa$ we expect to see a drop in the Ohmic dissipation rate as the number of sheets in the domain, and their intensity, declines.

Consider for example, the Ohmic dissipation of the field in the asymptotic limit $\kappa \gg 1$ given by (46) above. We have that

$$
\begin{aligned}
W_{\eta} & =\eta \int\left(\frac{2 \pi A}{L_{H}}\right)^{2}\left[\cos ^{2}\left(\frac{2 \pi x}{L_{H}}\right)+\sin ^{2}\left(\frac{2 \pi x}{L_{H}}\right)\right] d V \\
& =4 \eta\left(\frac{\alpha^{-} A}{\beta c_{H}}\right)^{2}=\frac{4 A^{2} \alpha^{-}}{\kappa} .
\end{aligned}
$$

This result shows that significant dissipation can be achieved via a multiplicity of (relatively weak) current layers, even for collisional resistivities. For example, if we conservatively assume that the numerator of (49) is order unity and take $\kappa$ $\simeq 10$ then a dimensionless dissipation rate $W_{\eta} \sim 10^{-1}$ is achieved. This translates to a strong power output of 4 $\times 10^{29} \mathrm{erg} / \mathrm{s}$. This output is achieved, not by resistive losses in the axial currents associated with the primary reconnection process, but by the Ohmic dissipation of relatively weak, Hall-induced separator currents distributed throughout the 
coronal volume. We know of no other merging solution based on collisional conditions which is capable of approaching such flare-like release rates.

There are however, two disclaimers to this result. In the first place the condition $\kappa \gg 1$ probably cannot be met if turbulent, enhanced resistivities are appropriate to the merging. Note that the oscillation length-scale (48) appropriate to $\kappa$ $\gg 1$ gives $L_{H} \simeq c_{H} \sim 10^{-6.5}$, corresponding to a rather short coronal length-scale of ten meters. The fact that the resultant current density $J=n e u^{*}$ corresponds to a proton sound speed of $u^{*}=10^{9} \mathrm{~cm} / \mathrm{s}$, a value appropriate to a very hot plasma of $10^{9} \mathrm{~K}$, already suggests that some form of enhanced, turbulent resistivity may be required to limit the current. ${ }^{4}$ If this is the case then $\kappa \gg 1$ probably cannot be realized and the primary, turbulently enhanced, current sheet will account for the bulk of the dissipation.

Second, even if collisional conditions are maintained, we must remember that the present analytic theory is based on the assumption of one-dimensional current sheets in shear flow velocity fields. The current sheet assumption holds good for conventional resistive merging, ${ }^{13}$ but as we shall see in the next section, and as described independently by other authors, ${ }^{3}$ we find evidence to suggest that the quasi-onedimensional current structure is undermined by the presence of strong Hall currents. The analytic treatment is also limited in its capacity to make predictions for head-on, Hall current reconnection (because $\beta=0$ turns off the Hall current independently of $c_{H}$ ). In the following section we explore the robustness of the idealized analytic solutions using a full numerical treatment of the dynamic merging problem.

\section{F. Summary}

The dynamic analysis of the merging problem confirms that, although Hall currents induce significant separator fields for $c_{H}>\eta$, they cannot change the character of the reconnection solution unless the much stronger condition $c_{H}^{2}>\eta(\kappa>1)$ is met. The steady-state description also suggests that in the asymptotic regime $\kappa \gg 1$, the solution develops an assemblage of secondary current layers on the size scale $\sqrt{\kappa \eta} \sim c_{H}$. Such layers clearly have the potential to add significantly to the dissipation provided by the primary reconnecting current sheet.

\section{HALL CURRENT RECONNECTION IN PERIODIC GEOMETRY}

\section{A. Introduction}

We now explore Hall current reconnection using a planar periodic code based on the systems (11)-(14). This code, first developed in Ref. 14, has been extensively used and tested in previous studies of planar reconnection. ${ }^{13,15}$ The present version includes Hall current and separator field contributions and differs from (11)-(14) only in the addition of a fluid viscosity (which is set at the level $\nu=\eta$ ). Accurate modeling of the Hall current does, however, place severe restrictions on the numerical time step since additional whistler wave modes are now present (see the Appendix).

A typical set of initial conditions given by

$$
\begin{aligned}
& \phi=\alpha_{0} \sin (\pi x) \sin (\pi y) / \pi, \\
& \psi=\beta_{0} \sin (\pi x) \sin (\pi y) / \pi+g_{0} \cos (\pi x) / \pi, \\
& W=Z=0,
\end{aligned}
$$

are allowed to evolve with time over a two-dimensional doubly periodic domain $(-1 \leqslant x, y \leqslant 1)$. These initial conditions determine a stagnation point flow near the origin that advects an initially one-dimensional magnetic disturbance field of amplitude $g_{0}$ toward the origin. This magnetic disturbance is amplified as it is washed inward, and eventually forms a current sheet aligned with the $y$-axis. Although the parameter $\alpha_{0}$ sets the flow amplitude-it mimics $\alpha$ in the previous analytic treatment $-\beta_{0}$ is best viewed (since pure magnetic annihilation of the planar field is impossible in this periodic geometry) as a parameter which governs whether the merging is head-on $\left(\beta_{0}=0\right)$ or sheared $\left(\beta_{0}>0\right)$.

In the runs that follow we choose the parameter $\beta_{0}$ to ensure that, for comparison with the analytic treatment, significant shear flows develop. Specifically we set $\beta_{0}=0.4$ and take $\alpha_{0}=-1$ and $g_{0}=0.03$. For all the runs presented in this paper we also fix $\eta=\nu=0.001$. The special case of head-on reconnection $\left(\beta_{0}=0\right)$ is discussed briefly in Sec. IV D.

Numerical experiments confirm that saturation of the current layer occurs if the flow field is not strong enough to localize the magnetic disturbance. ${ }^{14}$ This is due to the back pressure of the current sheet stalling the inflow, and can be avoided (at any given resistivity) simply by setting the disturbance field amplitude sufficiently small. To simplify the present analysis we take $g_{0}$ small enough to avoid saturation.

\section{B. Influence of the Hall term on the merging solution}

The analytic work of Sec. III suggests that Hall currents can affect the planar reconnection rate only if $\kappa \geqslant 1$. Figure 4 shows the perpendicular current generated by the planar merging field on the left and the planar currents generated by the Hall term on the right. Here the solution has been allowed to evolve until the primary reconnecting current sheet is fully developed (approximately 1.5 Alfvén times). The top plots, for $c_{H}=\eta$ corresponding to $\kappa=0.0002$, show little deviation from the $c_{H}=0$ case explored in Refs. 13 and 14 . The middle plots depict the regime $c_{H}>\eta(\kappa=0.02)$. As expected, the reconnecting current sheet (left plot) is largely unaffected despite the emergence of strong currents due to the induced separator field. Finally, the lower diagrams show the radical transformation of the central current sheet for $c_{H}^{2}>\eta(\kappa=3)$. In the final plot with $\kappa>1$, the classical "tombstone" geometry has evolved into a much sharper "arrowhead" and an extensive array of secondary, Hall current induced, sheets has developed due to the separator field.

How do these figures compare with the analytic theory developed by Craig and Watson? ${ }^{5}$ Let us begin by assuming that the open boundary, one-dimensional, steady-state theory, although not strictly applicable to dynamic merging in periodic geometries, should provide a crude model for the initial formation of the sheet. Then for $\kappa \gg 1$, we expect the development of periodic structures in the separator field of the form given in (46) with length scale $L_{H}$ along the inflow axis 

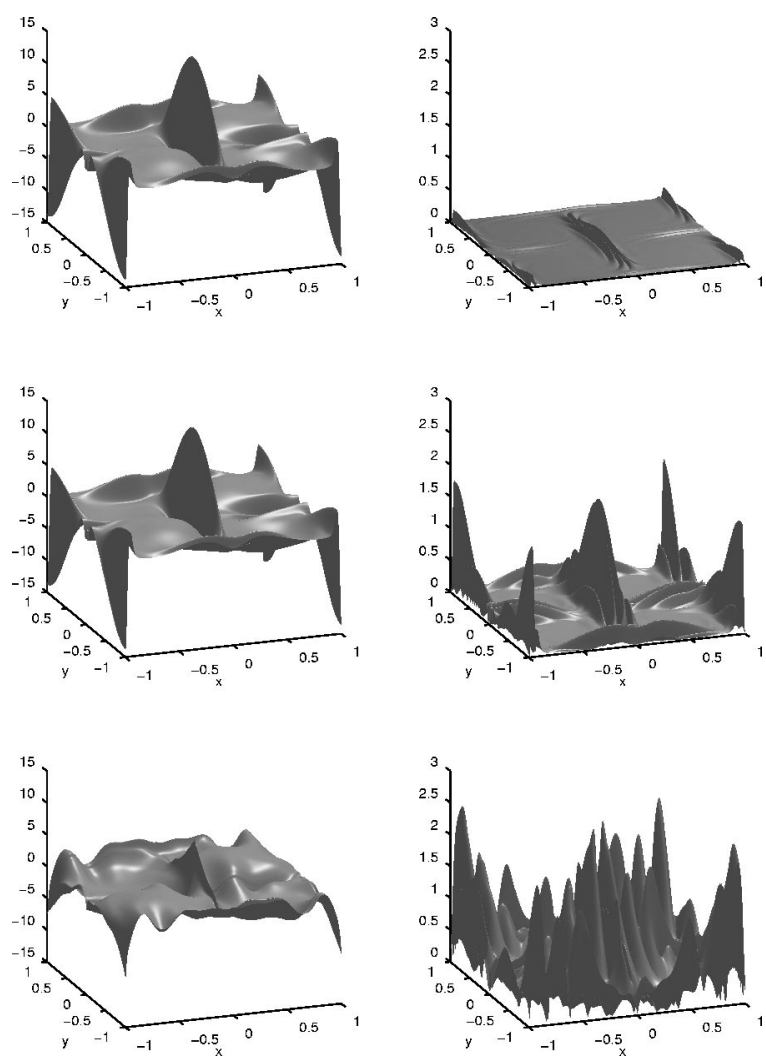

FIG. 4. Current in the $z$-direction $-\nabla^{2} \psi$ (left) and amplitude of the planar current $\sqrt{Z_{x}^{2}+Z_{y}^{2}}$ (right). The top plots are for $c_{H}=\eta=0.001 \quad(\kappa$ $=0.0002$ ), the middle use $\kappa=0.02$, while the bottom plots were obtained using $\kappa=3$.

given by (48). Figure 5 confirms that the predicted $Z$ field oscillations are well developed at the time of maximum dissipation (2.25 Alfvén times) for $\kappa=1$ and $\kappa=3$.

More quantitatively, in Fig. 6 we plot the computed oscillation length-scale for various values of $\kappa$. The solid line represents the steady-state analytic prediction (48), which is in good accord with the computed values for $\kappa>1$. This agreement is remarkable considering that the boundary conditions at $x= \pm 1$ will interfere with the oscillatory development if $\kappa$ is too large, a problem exacerbated by the relatively large resistivities used in numerical simulations.

There is mounting evidence in the literature that the Hall term causes magnetic merging to become less onedimensional and more Petschek-type (see, for instance, Ref. $3)$. The analytic model presented here, and in Craig and Watson, ${ }^{5}$ is severely limited by the choice of forms for $\mathbf{v}$ and B. Although our analytic model predicts a modification of traditional current sheet merging when a large Hall term is introduced, the current sheet still maintains its strict onedimensionality. It is unclear how to modify the model to address this shortcoming, however, it is a simple matter to explore these effects numerically. Indeed, we see clear evidence that if the disturbance field symmetries are violated (as they are in the periodic numerical code), then in the nonlinear regime the Hall term can severely modify the onedimensional current structure.

This effect is apparent in the lower left plot of Fig. 4 and is also clearly illustrated in Fig. 7, which shows two current
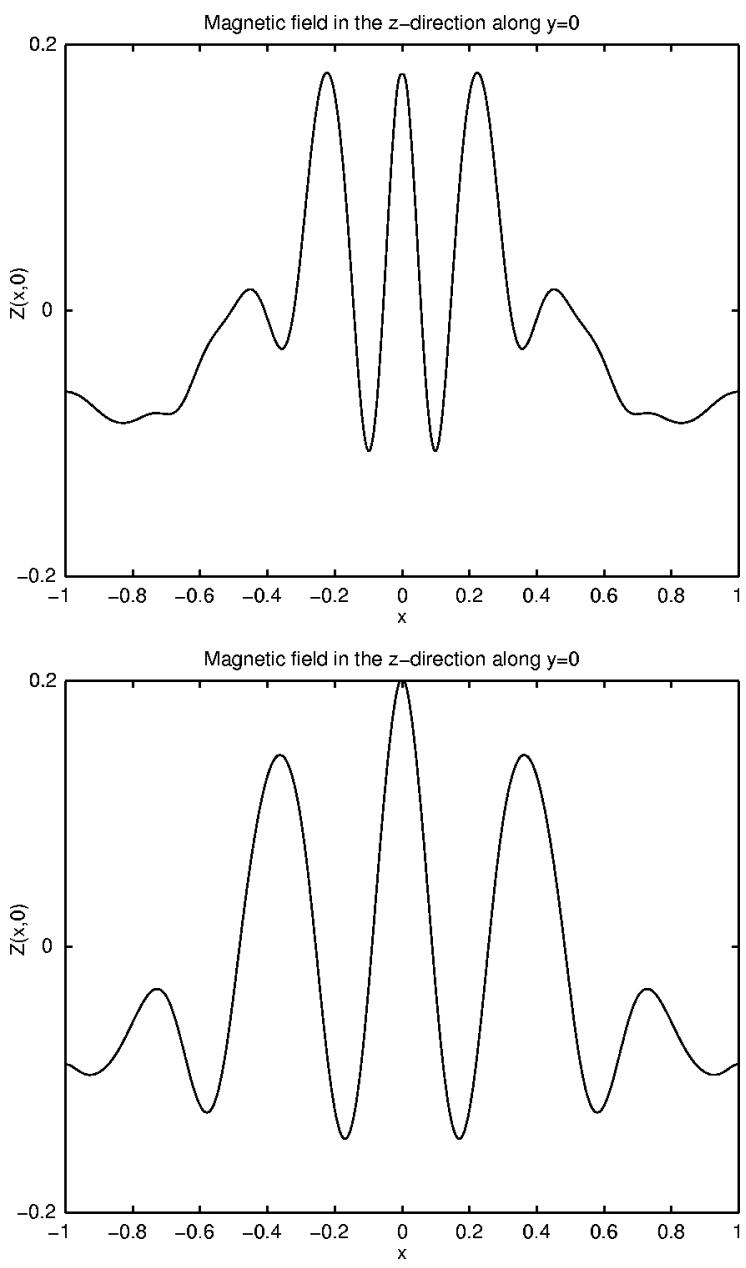

FIG. 5. Slices along the $x$-axis of the magnetic field in the $z$-direction taken at the time of maximum dissipation. The upper plot is for $\kappa=1$ and the lower for $\kappa=3$. Notice the variation in the periods of oscillation as predicted by (48).

sheets, with associated magnetic field lines and separatrix structures, at the time of maximum current (approx. 1.5 Alfvén times). The top plot is for a traditional resistive reconnection run with $\kappa=c_{H}=0$. Here the violation of the

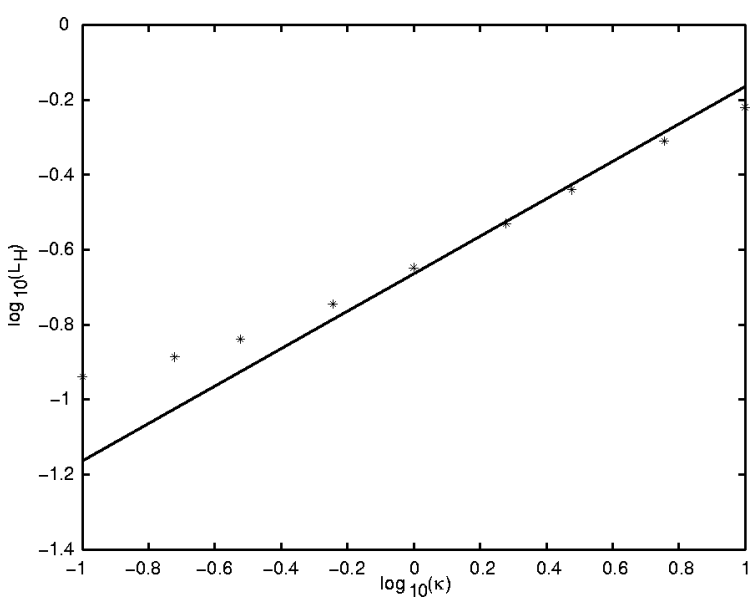

FIG. 6. Here we plot the length-scale associated with the $Z$ field against $\kappa$. The solid line is the the analytic prediction (48), while the asterisks indicate computed values. 

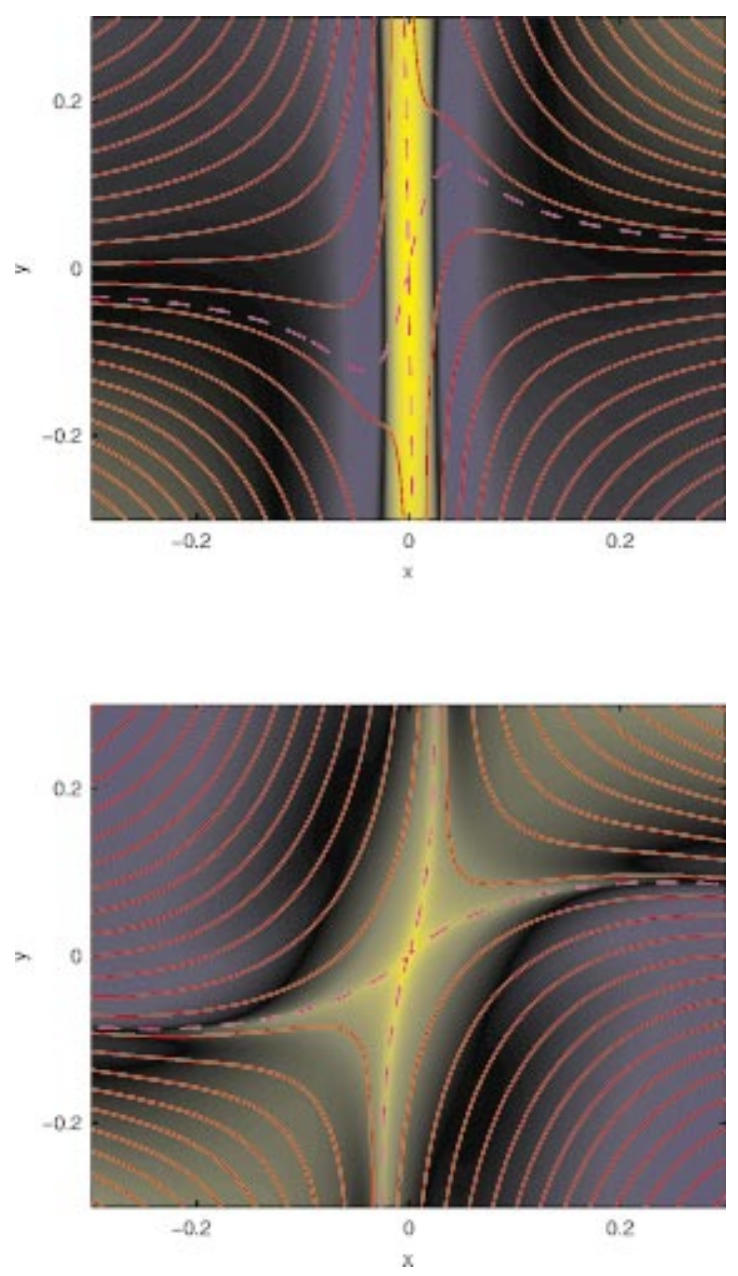

FIG. 7. (Color) Plots of magnetic field line structure (red), separatrix structure (purple) and $z$-direction current (shades of yellow and green) in the $z$ $=0$ plane. The top plot shows the classic long thin sheet when $\kappa=0$. The lower plot is for $\kappa=10$ and clearly shows well developed current structures along both $X$-point separatrices.

disturbance field symmetries induced by the periodic boundary conditions is unimportant and a long, thin quasione-dimensional current sheet is formed. This type of solution is described well by the analytic models, which accurately predict the sheet intensity and width and the very narrow angle of the field separatrices at the X-point. The lower plot is for the case of Hall current reconnection with $\kappa=10$. For this large value of the Hall term the violation of the symmetries has completely altered the picture. The separatrix angle is now much broader and the current sheet is no longer one-dimensional. In fact strong currents are now aligned along both separatricies, unlike the resistive MHD example in which current is only associated with one separatrix. This broadening of the $\mathrm{X}$-point angle and redistribution of the current seems to be an important consequence of the Hall term and it is unfortunate that it is not captured in the simple analytic model presented in Sec. III. Several authors ${ }^{1,3,6}$ have observed these phenomena and have suggested that the inclusion of the Hall term makes the reconnection more Petschek-type, although we should stress that the reconnection mechanism is quite different to that of Petschek.

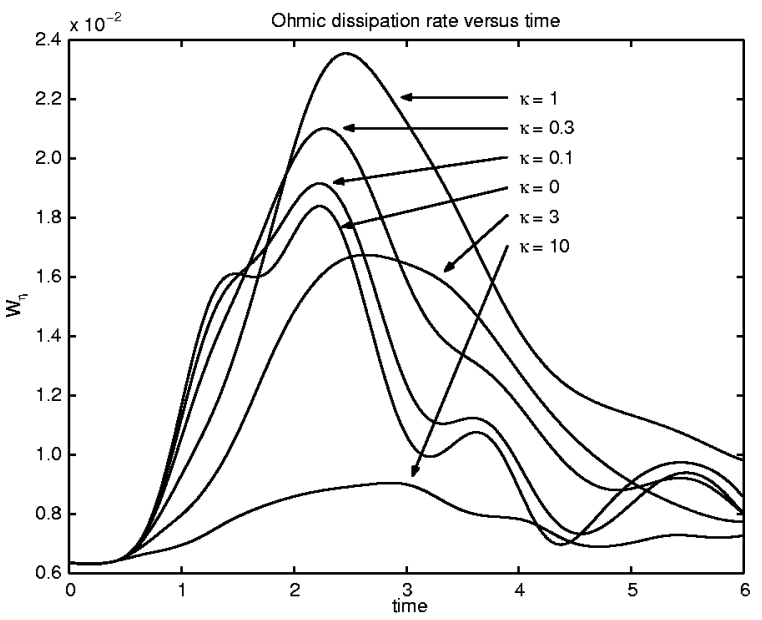

FIG. 8. Traces of the Ohmic dissipation (51) integrated over the entire domain for various values of $\kappa$. The $\kappa=0$ case shows the cyclic dissipation due to the formation and dissipation of current sheets due only to the planar merging. As $\kappa$ increases, the Hall current sheets compound the overall dissipation. However, for $\kappa>1$ the structure of the planar merging is lost, and at the same time the number of Hall sheets diminishes leading to lower dissipation rates

\section{Ohmic decay rates as a function of $\kappa$}

As already mentioned, although the reconnecting current sheet generated by the planar merging becomes less dominant for larger values of $\kappa$, see Fig. 4, the development of secondary current sheets, due to the Hall-induced separator field, can lead to enhanced dissipation over the traditional $c_{H}=0$ models. Figure 8 shows traces of the computed Ohmic dissipation,

$$
W_{\eta}=\eta \int J^{2} d V=\eta \int\left(Z_{x}^{2}+Z_{y}^{2}+\left(\nabla^{2} \psi\right)^{2}\right) d V
$$

over time for various values of $\kappa$. It is clear from these plots that moderate values of $\kappa$ allow for enhanced dissipation, especially at later times when the Hall current layers are well developed. However, there seems to be an optimum level for the Hall current contribution, around $\kappa=1$, that preserves most of the primary current sheet, while at the same time allowing extra dissipation from the perpendicular separator field.

Note that, for very large values of $\kappa$, the total Ohmic dissipation is reduced. This effect is consistent with the result (49) of the previous section,

$$
W_{\eta} \simeq \frac{4 A^{2} \alpha^{-}}{\kappa} \quad(\text { for } \kappa>1) .
$$

A marked decline in the maximum dissipation is evident in Fig. 8 for $\kappa>1$ and it seems clear that the asymptotic regime is entered even for moderate values of $\kappa$. Note also that this expression provides a plausible estimate of the actual level of Ohmic dissipation found in the computations: taking $\alpha^{-}$ $\simeq 0.84, A=0.15$ (see Fig. 5) and $\kappa=3$ yields $W_{\eta} \simeq 0.025$, corresponding to dimensional rate of $10^{29} \mathrm{erg} / \mathrm{s}$. This estimate, although clearly in the right ballpark according to Fig. 
8 , is expected to be accurate only in the asymptotic regime of large $\kappa$ and only if the dissipation is achieved through quasione-dimensional current layers.

\section{Symmetries in Hall current merging}

In the simple analytic model presented in Sec. III and in most of the numerical results presented in this section the addition of Hall current effects has lead to a decrease in the reconnection rate. Most previous studies ${ }^{1,3,6}$ have reported that the inclusion of Hall effects can result in a dramatic increase in the reconnection rate. It is not difficult to trace this discrepancy to the symmetries of the problems being studied. In this work we have considered the case of shear flow reconnection, in which impacting flux surfaces are not constrained to the fourfold symmetry of head-on merging. Conversely, most other authors have examined highly symmetric head-on reconnection configurations.

Why does the reconnection geometry have such a dramatic effect? This behavior can be explained by returning to Eq. (13) and rewriting it in the form

$$
\psi_{t}+\left[\psi, \phi-c_{H} Z\right]=\eta \nabla^{2} \psi .
$$

It is now clear that in the absence of resistivity the magnetic field $\psi$ is advected by the modified stream function,

$$
\phi_{e}=\phi-c_{H} Z \text {, }
$$

where $\phi_{e}$ can be associated with the stream function for the electron fluid [this follows-after nondimensionalizingfrom the definition $\mathbf{J}=n e\left(\mathbf{v}_{\mathbf{i}}-\mathbf{v}_{\mathbf{e}}\right)$ where $\mathbf{v}_{\mathbf{i}}$ is the ion velocity and $\mathbf{v}_{\mathbf{e}}$ is the electron velocity]. In other words in Hall current reconnection the field is tied to the electrons and not the ions. Obviously the perpendicular field $Z$ can influence the reconnection rate by altering the inflow velocities in the vicinity of the reconnection region, i.e., if $Z$ acts to increase the inflow speed into the sheet then the reconnection rate is increased and vice versa. When $Z=Z(x)$, as is assumed in our analytic model, the perpendicular field leaves the inflow velocity $u_{e}$ $=\partial \phi_{e} / \partial y=\partial \phi / \partial y$ unchanged, and so it is not expected to lead to an increase in the reconnection rate. In fact the $Z$ field leads to a decrease in the shear flow across the sheet, which acts to slow the reconnection rate by slowing the exhaust flows. Traditional head-on symmetries do not place this restriction on the functional form of $Z$, and so two-dimensional $Z$ fields can develop that enhance the inflow speeds and the reconnection rate. This is confimed by numerical simulations for head-on reconnection $\left(\beta_{0}=0\right)$.

A comparison of head-on and sheared reconnection shows that for head-on configurations the separator field again mimics the build-up of the axial reconnection current. Significant differences emerge, however, in the regime $c_{H}^{2}$ $\simeq \eta$, where the reconnection rate is affected by the separator field. The extra symmetry constraints implied by head-on merging mean that the induced separator component must develop a two-dimensional structure. This leads to significant departures from the case of sheared reconnectioncomputations confirm that there is now a reduction of the Ohmic losses with increases in the Hall coefficient $c_{H}$, but an increase in the reconnection rate. These differences, which have no echo in classical resistive computations, ${ }^{13}$ suggest that Hall current reconnection is far more sensitive to the initial and boundary conditions imposed on the merging problem.

\section{CONCLUSIONS}

We have explored the role of Hall currents in planar, dynamic reconnection solutions using two distinct approaches. The first approach, a dynamic analytic treatment based on the assumption of quasi-one-dimensional current layers, confirms that the condition $c_{H}>\eta$ is required for the emergence of a significant separator component in the reconnecting magnetic field. However, in line with the earlier steady-state treatment of Craig and Watson, ${ }^{5}$ a much stronger condition $c_{H}^{2}>\eta$, or equivalently $\kappa>1$, is required if the burgeoning separator field is to influence the reconnection rate and significantly alter the morphology of the reconnecting planar field. The analytic treatment also provides a graphic illustration of how planar magnetic field disturbances, advected by simple stagnation point flows, can generate large axial currents, which through the mechanism of the Hall current, induce large amplitude separator fields.

In the second approach, outlined in Sec. IV, a series of numerical simulations were performed, based on shear flow reconnection in a closed, periodic reconnection geometry. These computations reinforce and considerably extend the results of the analytic study. In particular, the morphology of the reconnection solution changes dramatically for $\kappa>1$, as illustrated, for example, in Fig. 7. A key question, therefore, is how the strong separator field affects the reconnection rate in the critical regime $c_{H}^{2} \rightarrow \eta$. The answer seems to depend on the details — in particular the symmetries — of the merging simulation.

For instance, in this paper we have concentrated on the general case of sheared reconnection. Although increasing the Hall parameter generally slows down the reconnection rate, the resistive dissipation can, for $\kappa \sim 1$, increase due to the emergence of multiple secondary current layers associated with the separator field. More specifically a whistler wave (see Figs. 3 and 5) is responsible for setting up global oscillations of length-scale $L_{H} \simeq \sqrt{\kappa \eta}$ in the separator field. For the special case of head-on reconnection, however, the separator field is constrained by the symmetry of the merging to vanish at all points on the inflow axis; the net result in this case is an enhancement in the reconnection rate but a decrease in the global Ohmic dissipation. This sensitivity to the details of the reconnection geometry appears to be a feature of Hall current reconnection that has no analog in classical resistive merging. Clearly, in view of the rich structure present in Hall current reconnection, some care should be exercised in making generalized claims based on specific Hall current simulations.

\section{APPENDIX: TIME STEP LIMIT FOR HALL MHD}

In the explicit scheme we employ, the presence of whistler modes places severe restrictions on the numerical time step. Specifically, the CFL limit on the advective time step is of the form, 


$$
\Delta t<\frac{\Delta x}{v_{p}},
$$

where $v_{p}$ is the maximum phase velocity over the mesh for waves in the system. For purely resistive, incompressible MHD the relevant phase velocity is $v_{p}=v+v_{A}$, where $v$ is the local plasma velocity and $v_{A}$ is the local Alfvén speed. However, the equations of incompressible Hall MHD admit another type of wave, the so-called whistler wave. These waves are more troublesome computationally than Alfvén waves because they are dispersive, i.e., their phase velocities depend on the wave number $k$.

To see this we begin with the primitive Hall MHD equations and neglect diffusive effects. We linearize about a static equilibrium with a constant field $\mathbf{B}_{0}$, so that

$$
\mathbf{v}=\mathbf{v}_{1}, \quad \mathbf{B}=\mathbf{B}_{0}+\mathbf{B}_{1} .
$$

The governing equations are now given by

$$
\begin{aligned}
& \rho \dot{\mathbf{v}}_{1}=\nabla \times \mathbf{B}_{1} \times \mathbf{B}_{0}, \\
& \dot{\mathbf{B}}_{1}=\nabla \times\left(\mathbf{v}_{1} \times \mathbf{B}_{0}\right)-c_{H} \nabla \times\left(\nabla \times \mathbf{B}_{1} \times \mathbf{B}_{0}\right) .
\end{aligned}
$$

If we look for Fourier mode wave solutions of the form,

$$
\begin{aligned}
& \mathbf{v}_{1}=\hat{\mathbf{v}}_{1} e^{i(\mathbf{k} \cdot \mathbf{x}+\omega t)}, \\
& \mathbf{B}_{1}=\hat{\mathbf{B}}_{1} e^{i(\mathbf{k} \cdot \mathbf{x}+\omega t)},
\end{aligned}
$$

and make use of the divergence free nature of $\mathbf{v}$ and $\mathbf{B}$, we find the perturbed field vector $\hat{\mathbf{B}}_{1}$ must satisfy

$$
i\left(\omega-\frac{\left(\mathbf{k} \cdot \mathbf{B}_{0}\right)^{2}}{\rho \omega}\right) \hat{\mathbf{B}}_{1}=c_{H}\left(\mathbf{k} \cdot \mathbf{B}_{0}\right) \mathbf{k} \times \hat{\mathbf{B}}_{1} .
$$

Letting $\mathbf{k}=k \hat{\mathbf{x}}, \mathbf{B}_{0}=B_{0} \hat{\mathbf{x}}$, and $\hat{\mathbf{B}}_{1}=\left(0, \hat{B}_{1 y}, \hat{B}_{1 z}\right)$ (consistent with the divergence free conditions) we find $\hat{B}_{1 y}$ and $\hat{B}_{1 z}$ must satisfy the system

$$
\left(\begin{array}{cc}
i\left(\omega-\frac{k^{2} B_{0}^{2}}{\rho \omega}\right) & c_{H} k^{2} B_{0} \\
-c_{H} k^{2} B_{0} & i\left(\omega-\frac{k^{2} B_{0}^{2}}{\rho \omega}\right)
\end{array}\right)\left(\begin{array}{c}
\hat{B}_{1 y} \\
\hat{B}_{1 z}
\end{array}\right)=0 .
$$

Setting the determinant of this system to zero we find the dispersion relation

$$
\omega= \pm \frac{c_{H} k^{2} B_{0} \pm \sqrt{c_{H}^{2} k^{4} B_{0}^{2}+\frac{4 k^{2} B_{0}^{2}}{\rho}}}{2} .
$$

Hence the system admits four circularly polarized waves (a modified Alfvén wave and a whistler wave, both traveling to the left or right).

In the large $c_{H}$ limit $\omega \simeq c_{H} k^{2} B_{0}$ and we find

$$
v_{p}=\frac{\omega}{k}=c_{H} k B_{0} .
$$

The relevant CFL condition therefore becomes

$$
\Delta t<\frac{\Delta x}{c_{H} k B_{0}}=\frac{\Delta x^{2}}{\pi c_{H} B_{0}},
$$

where we have assumed that the maximum wave number on the mesh corresponds to point-to-point oscillations. Clearly this new time step limit is far more severe than that for ordinary Alfvén waves, and is very sensitive to increases in resolution.

${ }^{1}$ J. Birn, J. F. Drake, M. A. Shay et al., J. Geophys. Res. 106, 3715 (2001).

${ }^{2}$ E. R. Priest and T. G. Forbes, Magnetic Reconnection MHD Theory and Applications (Cambridge University Press, Cambridge, 2000).

${ }^{3}$ A. Bhattacharjee, Z. W. Ma, and X. Wang, J. Geophys. Res. 104, 14543 (1999).

${ }^{4}$ Y. E. Litvinenko and I. J. D. Craig, Astrophys. J. 544, 1101 (2000).

${ }^{5}$ I. J. D. Craig and P. G. Watson, Sol. Phys. 214, 131 (2003).

${ }^{6}$ A. Bhattacharjee, Z. W. Ma, and X. Wang, Phys. Plasmas 8, 1829 (2001).

${ }^{7}$ L. Spitzer, The Physics of Fully Ionized Gases, 2nd ed. (Interscience, New York, 1962).

${ }^{8}$ I. J. D. Craig and S. M. Henton, Astrophys. J. 450, 280 (1995).

${ }^{9}$ E. R. Priest, V. S. Titov, R. E. Grundy, and A. W. Hood, Proc. R. Soc. London 456, 1821 (2000).

${ }^{10}$ I. J. D. Craig and R. B. Fabling, Phys. Plasmas 5, 635 (1998).

${ }^{11}$ I. J. D. Craig and A. N. McClymont, Astrophys. J. 510, 1045 (1999).

${ }^{12}$ B. U. Ö. Sonnerup and E. R. Priest, J. Plasma Phys. 14, 283 (1975).

${ }^{13}$ J. Heerikhuisen, I. J. D. Craig, and P. G. Watson, Geophys. Astrophys. Fluid Dyn. 93, 115 (2000).

${ }^{14}$ I. J. D. Craig and P. G. Watson, Astrophys. J. 516, 924 (1999).

${ }^{15}$ P. G. Watson and I. J. D. Craig, J. Geophys. Res. 106, 15735 (2001). 